\title{
Elliott, Kevin C. (2017), A Tapestry of Values: An Introduction to Values in Science, New York: Oxford University Press, 224pp, ISBN 9780190260804.
}

Elliott's book intends to provide an "accessible" and "effective" introduction to the role of values in science (p. xi). The book consists of seven chapters plus the 'Preface' and 'Conclusion', structured in the way I will explain below. It also includes annotated references at the end of each chapter, numerous tables displaying different theses at a glance, and a list of questions for further reflection at the end of the book. These tools prove to be very helpful to readers and potential course instructors.

Any discussion of the roles of values in science must begin with an understanding of these two concepts. Elliott does not define "science", but it is clear that the term is meant to cover the research activities (pure or applied) that are currently recognized as scientific. The notion of "value" is instead explicitly explained as "something that is desirable or worthy of pursuit" (p. 11). But in the very next sentence (and elsewhere) it is clear that values in this book are rather what people value, what people regard as desirable: "for example, scientists typically value accurate predictions, clear explanations [...] health, economic growth" (p. 11). As this partial list shows, there are different kinds of values that matter to scientists: some of them are epistemic, that is, they contribute to knowledge (accurate predictions, logical consistency, etc.), others are non-epistemic, and can be described as ethical, political, religious, aesthetical, personal or economic, as the case may be.

The book explores (1) the descriptive question of whether and how non-epistemic values both influence choices made by scientists and are in turn supported (knowingly or not) by these choices, and (2) the normative question of whether and under what conditions should non-epistemic values influence science. Elliott's answer to the first part of both questions is positive: non-epistemic values do systematically and unavoidably influence science—so that there is no real room for requiring that science be value-free; that just would not be science as we know it - and, under certain conditions, they also should influence it. His overall answer to question (1) is justified and illustrated in Chapters 2-6. His overall answer to question (2) is made explicit in the 'Preface', 'Conclusion', Chapters 1 and 7, and can be found in various places throughout the book. I will discuss the two questions in turn. 
Elliott's assumption is that "the roles of values can be analytically disentangled from other aspects of scientific reasoning" (p. 165). This is crucial: if the distinction between value and non-value questions in science is blurred (as probably some philosophers would maintain), then we are not able to critically examine the influence of values on science, so we are not able to answer question (2). But we need to do some spadework to unearth these influences, which are sometimes deliberate (e.g., when a scientist is motivated by ethical convictions or by financial incentives), but often subtle and only seen a posteriori.

So how and when do values (from now on understood as non-epistemic) inform science? One part of the novelty and merit of Elliott's work is to ignore the "sidelines" of research, where value discussions have traditionally taken place (e.g., faking data, plagiarism, research on human or animal subjects), and focus instead on the ways that values impact on the core of what he calls "scientific reasoning”. Another part of his merit is to finely and systematically identify several (five, if we count by chapters) of these core activities, where scientists are called on to make decisions in which appeal to values is unavoidable: decisions as to what research topics one should prioritize, how to study them (which methodologies should be employed), which specific aims one should have (which results one should be looking for), how one should respond to uncertainty (both in the lab, so to speak, and in front of affected parties), and how to frame and otherwise communicate results to the public.

For each of the five core activities, Elliott illustrates the value influences that may be at work with helpful study cases. But what makes them all part of "scientific reasoning"? The reader might get an initial impression that Elliott has simply put together a list of relevant, but otherwise unrelated, moments of scientific activity. This is not the case. The book comes into its theoretical nucleus as Elliott claims: "Different sorts of values are relevant when scientists adopt different cognitive attitudes" (p. 73). Epistemic values are typically the ones to draw on when deciding whether a theory is true or reliable-that is, when deciding whether to believe it or not. But the study cases concern other cognitive attitudes, which are very much a scientist's daily routine: pursuing a certain theory, making hypotheses, developing a theory further, looking for objections, asking for further evidence, and more practical ones like publicly defending a theory, and in general communicating about one's findings. While this might again look like an unprincipled list, the common theme is that the truth or likelihood of a given theory alone cannot settle questions as to whether to adopt these attitudes. In forming any of these attitudes, scientists must be 
responsive to non-epistemic reasons, such as the ethical, etc. values that would be promoted or undermined.

Elliott repeatedly points out that allowing value influences on these attitudes does not amount to wishful thinking - this is when my belief that $p$ (or my rejection of $p$ ) is partially grounded on my desire that $p$ be true (or false). Since the attitudes listed above go beyond belief, they are not even exposed to the problem of wishful thinking. Still, the influence of non-truth-related reasons may threaten their good standing as cognitive attitudes, that is, as attitudes aimed at serving the cause of knowledge. Such tension is most clearly illustrated in two adjacent sections of Chapter 5.

One concerns the issue of how to phrase and communicate one's results to the public. Elliott usefully distinguishes between three approaches (pp. 87-92): "clean-hands" science, by simply sticking to the data and suspending any overall judgment, serves objectivity, but does not do any service to policymakers; "modified clean-hands" science, by making explicit but qualified judgments, favours the cause of knowledge-because the qualifications prevent misunderstandings-but those same qualifications are easy to get lost or may confuse policy-makers; finally, the "advocacy" approach, by preferring simple, stark judgments, may well promote values like public health, but undermines the actual (and perceived) objectivity of science. The tension is clear: in each case the epistemic benefits of an approach tend to be inversely proportional to its social benefits.

The subsequent section concerns an even more difficult question: when is evidence enough to draw a conclusion (pp. 92-100)? While it might sound surprising to allow non-epistemic reasons to weigh in at this stage, the socalled argument from inductive risk (by Heather Douglas) suggests that when the risk attending an erroneous conclusion is significant enough, scientists may (and should) accordingly adjust the standards and amount of evidence required: great risks may justify relying on weaker or limited evidence in favour of a conclusion (e.g., that dioxin causes cancer). This generates an obvious tension: asking for less evidence might have social benefits, but also makes one's conclusion less likely to be true. Elliott's strategy is to somewhat alleviate the tension in two ways (pp. 97-100): standards of evidence should be settled in advance by groups of scientists according to agreed guidelines (to reduce arbitrariness in making such decisions), and they should be adjusted depending on who the stakeholders are (other researchers, policy-makers, the judiciary, citizen groups, etc.). What is not discussed, though, is whether these 
strategies may also compensate the epistemic shortcomings of an approach such as Douglas's (I will come back to this below).

Regarding the book's second question-whether and under what conditions should non-epistemic values influence science-Elliott usefully sums up two main justifications for bringing values explicitly to bear on scientific reasoning (pp. 13-14, 167): (1) As he shows in each chapter, many central choices made by scientists (before, during, and after the "discovery process") end up supporting some non-epistemic values over others, so scientists had better be ready to be held accountable, at least so far as some responsibility does fall on them; (2) In being guided by (the right kind of) non-epistemic values, scientists can serve society over and beyond their contribution to knowledge. But this raises the question of just which values should be allowed such influence. Elliott does not provide a list of values, but rather recommends three conditions as necessary (pp. x, 10, 14-15, 105-107, 170-172): scientists should be transparent about the values they (intentionally or not) promote; these values should represent fundamental ethical and social priorities; stakeholders and other segments of society should be involved in the process in appropriate ways (partly specified in Chapter 7). Certain value influences-for example, corporate interests-thus need not be inappropriate because of the nature of the values themselves, but because and when they are kept concealed or undermine the involvement of other stakeholders.

But why, exactly, these conditions and not others? I will end by asking a question that is relevant in this respect, but which Elliott regrettably does not take up: does the right kind of engagement with the right kind of non-epistemic values also tend to promote scientific or epistemic goals? The case studies examined in Chapter 7 do not speak clearly in this sense; for example, it is not said whether AIDS research was in fact significantly improved, in terms of accuracy and reliability, by the interaction of researchers, policy-makers and community groups. Elliott very briefly considers one facet of this question by suggesting that communities not only voice legitimate non-epistemic demands or values, but may also contribute precious "local knowledge" or "traditional ecological knowledge" (pp. 51, 144-145). But it is not clear how decisive this epistemic contribution is in general. Also, the book does open with the success story of how Theo Colborn's commitment to environmental values and public health led her to discover the phenomenon of "endocrine disruption" by certain chemicals (pp. 5-7). However, it seems difficult to read her case as pointing to something systematic. Colborn might simply be one of many scientists who happen to be 
both decent persons and talented researchers. And the final chapter includes the story of biologist Barry Commoner, who joined his ethical and political commitment with his scientific research. But the moral in his case is that "scientists can incorporate values in their work in many different ways without sacrificing the quality of their science" (p. 165). My question here is whether non-epistemic values may rather improve the quality of scientific research.

On the other hand, Elliott provides plenty of evidence for a negative claim, namely that engagement with, or the secret influence of, mistaken or partial non-epistemic values tends to undermine scientific goals_-in other words, nonepistemic error breeds epistemic error. For example, arguably implicit sexist beliefs about the 'proper' roles of men and women may have misled generations of anthropologists and evolutionary biologists into accepting false 'man-the-hunter' models of human evolution (Chapter 4). Another clear example is the way that bad or at least unreliable science often results from corporate interests, which rarely if ever satisfy Elliott's conditions for appropriate value influence. He offers several cases of such influence throughout the book: the accuracy of toxicological analysis - whether it concerns factory waste or for example new materials-is typically jeopardized by the interests of the relevant industries (pp. 48-50, 9293); the novelty of drug research tends to be thwarted by patenting policies which allow pharmaceutical companies to patent (and thus earn a profit from) new drugs that are only minimally different from others (pp. 35-36); medical researchers may be drawn to 'discover' non-existent diseases (p. 55); and so forth.

An asymmetry thus emerges from Elliott's own treatment: the correlation between unacceptable values and bad science seems much stronger than that between acceptable values and good science. Otherwise said, chances are that science influenced by acceptable values would have been good science regardless, while science influenced by unacceptable values turns out (often enough) to be bad science. If this diagnosis is correct, it might incline one towards wishing for a science that is as free from non-epistemic values as possible. But this is a wish that Elliott himself repeatedly dismisses as both questionable and practically hopeless. Elliott should therefore pursue either or both of these strategies: do something to correct the impression of the asymmetry above and/or show how, even if there is such an asymmetry, one should still favour value-influenced over value-free science.

Going forward, the book can be a useful reference point to think about current debates (within and outside philosophy) about the nature, value, and limitations of expertise. Elliott does not certainly favour anything like mistrust of scientists. 
Still, the notion that forms of involvement with society should be actively pursued, as well as his rejection of the "deficit" thesis that people's opposition to science is only caused by their ignorance (pp. 169-170), do suggest that scientists should do more to earn their social status than is normally acknowledged.

I would recommend the interested reader to explore more in depth these questions, as well as the issue of inductive risk above, in two collections recently co-edited by Elliott: Current Controversies in Values and Science (with Daniel Steel, Routledge, 2017) and Exploring Inductive Risk: Case Studies of Values in Science (with Ted Richards, Oxford University Press, 2017).

\section{Francesco Orsi}

Institute of Philosophy and Semiotics, University of Tartu

Jakobi 2,

Tartu 51005, Estonia

E-mail: francesco.orsi@ut.ee 